Note

\title{
Oral Administration of Freeze-Dried Kefir Reduces Intestinal Permeation of and Oral Sensitization to Ovalbumin in Mice
}

\author{
Chisato Umeda, ${ }^{1}$ Kei Sonoyama,,${ }^{1, \dagger}$ Natsu Yamaguchi, ${ }^{1}$ Ryo Saito, ${ }^{1}$ \\ Keiko Akashi, ${ }^{2}$ Hidemasa Motoshima, ${ }^{2}$ and Jun Kawabata ${ }^{1}$ \\ ${ }^{1}$ Laboratory of Food Biochemistry, Division of Applied Bioscience, Graduate School of Agriculture, \\ Hokkaido University, Sapporo 060-8589, Japan \\ ${ }^{2}$ Research Center, Yotsuba Milk Products Co., Ltd., Kitahiroshima 061-1264, Japan
}

Received September 17, 2004; Accepted October 27, 2004

\begin{abstract}
An increase in plasma ovalbumin concentrations after intragastric administration of ovalbumin was suppressed by concomitant freeze-dried kefir in BALB/c mice. Serum levels of ovalbumin-specific immunoglobulin $\mathbf{G}$ and proliferation of splenic mononuclear cells in mice immunized orally with ovalbumin were suppressed by feeding freeze-dried kefir. We propose that kefir reduces intestinal permeation of food antigen, which contributes to suppression of oral sensitization.
\end{abstract}

Key words: kefir; ovalbumin; gastrointestinal permeation; oral sensitization; $\mathrm{BALB} / \mathrm{c}$ mice

The gastrointestinal tract constitutes the largest interface between animals and their external environment. As a barrier, it prevents the penetration of harmful entities such as food antigens. Hyperpermeability of this barrier is believed to contribute to the pathogenesis of several gastrointestinal disorders, including food allergy. Therefore it is rational to strengthen the barrier function and to inhibit the penetration of food antigens in the gastrointestinal tract for prevention of food allergy. With these considerations in mind, we have been pursuing studies involving the impact of food substances on the intestinal barrier to inhibit permeation of food antigens. ${ }^{1-4)}$

Kefir is a beverage made from milk fermented with a complex mixture of lactobacilli, lactococci, leuconostocs, and yeasts. Because consumption of yogurt and lactic acid bacteria (i.e., probiotics) has been reported to improve or prevent allergic symptoms, ${ }^{5-10)}$ kefir was expected to have a similar impact on allergy. The present study aimed to examine whether consumption of kefir affects the intestinal penetration of and oral sensitization to food antigens. Reduced sensitization to food antigen should result in prevention of food allergy.

In this study, kefir was prepared by the Research Center of Yotsuba Milk Products Co., Ltd., as follows: Kefir grain was maintained by subculture in reconsti- tuted skim milk. The propagated-kefir grain was strained with chicken wire, and the filtrate was then used as a starter. Kefir was prepared by the addition of approximately $1 \%(\mathrm{w} / \mathrm{v})$ of the starter to the reconstituted milk $(3 \%(\mathrm{w} / \mathrm{v})$ of fat and $9.5 \%(\mathrm{w} / \mathrm{v})$ of solid-non-fat), cultivated at $22{ }^{\circ} \mathrm{C}$ overnight, and then cooled and matured at $5{ }^{\circ} \mathrm{C}$. The resultant kefir was freeze-dried and subjected to animal experiments. Six-week-old female BALB/c mice were purchased from Japan Charles River (Yokohama, Japan). They were maintained in a temperature-controlled $\left(23 \pm 2{ }^{\circ} \mathrm{C}\right)$ room with a dark period from 19:00 to 5:00 h, and acclimatized with free access to tap water and a purified diet prepared according to AIN-93G. ${ }^{11)}$ After 1 week of acclimatization, the mice were deprived of food for $12 \mathrm{~h}$ and then intragastrically administered either $0.3 \mathrm{ml}$ of PBS containing $20 \mathrm{mg}$ of ovalbumin (OVA, Grade V, Sigma, MO, U.S.A.) or the same solution supplemented with $5 \mathrm{mg}$ of freeze-dried kefir. Blood samples were collected from the tail vein at $0,30,60,120$, and 180 min after feeding, and the plasma OVA concentration was measured by sandwich ELISA, as previously described. ${ }^{1)}$ In a separate experiment, 5week-old female BALB/c mice were purchased from Japan Charles River and maintained as described above. After 1 week of acclimatization, the mice were fed either the AIN-93G diet as a control diet or that diet supplemented with freeze-dried kefir $(50 \mathrm{~g} / \mathrm{kg}$ diet $)$ for 7 weeks, and $0.2 \mathrm{ml}$ of PBS containing $0.1 \mathrm{mg}$ of OVA was intragastrically administered to all animals every day. Blood samples were obtained from the tail vein at weekly intervals and put through ELISA for measurement of OVA-specific immunoglobulin $\mathrm{G}$ ( $\mathrm{IgG}$ ) concentration, as previously described. ${ }^{12)}$ On the last day of the experiment, the mice were euthanized, and splenic mononuclear cells were isolated using NycoPrep (AxisShield, Oslo, Norway) according to the suggested protocol, and subjected to proliferation assay. The cells were cultured with specific antigen $(100$ or $1,000 \mu \mathrm{g} / \mathrm{ml}$ OVA) or mitogen $(10 \mu \mathrm{g} / \mathrm{ml}$ phytohemagglutinin-P,

\footnotetext{
$\dagger$ To whom correspondence should be addressed. Fax: +81-11-706-2496; E-mail: ksnym@chem.agr.hokudai.ac.jp Abbreviations: BrdU, bromodeoxyuridine; IgG, immunoglobulin G; OVA, ovalbumin; PHA-P, phytohemagglutinin-P
} 
PHA-P) for $72 \mathrm{~h}$ in flat-bottom 96-well plates at $1 \times 10^{5}$ cells/well in RPMI1640 supplemented with $10 \%$ heat-inactivated fetal calf serum, $100 \mathrm{U} / \mathrm{ml}$ penicillin, $100 \mu \mathrm{g} / \mathrm{ml}$ streptomycin, $50 \mu \mathrm{g} / \mathrm{ml}$ gentamycin, and $50 \mu \mathrm{M}$ 2-mercaptoethanol. The cultures were pulsed with bromodeoxyuridine (BrdU) $72 \mathrm{~h}$ later and harvested $6 \mathrm{~h}$ after that. Incorporated BrdU was measured using Cell Proliferation ELISA BrdU (Roche Diagnostics, Tokyo, Japan) according to the suggested protocol. An unpaired $t$-test was used to compare mean values. The animal experiments in this study were approved by the Hokkaido University Animal Use Committee, and the animals were maintained in accordance with the guidelines for the care and use of laboratory animals at Hokkaido University.

The plasma concentration of OVA in the mice was highest $30 \mathrm{~min}$ after administering OVA, and decreased thereafter (Fig. 1). The levels at $30 \mathrm{~min}$ were significantly lower in mice administered freeze-dried kefir together with OVA than in those administered OVA alone, suggesting suppression of intestinal penetration of OVA by kefir. Although further experiments are needed to identify the constituent responsible for the suppression of OVA penetration, one of the candidates is kefiran, an extracellular polysaccharide produced by Lactobacillus kefiranofaciens in kefir grain. ${ }^{13)}$ Kefiran has physicochemical properties such as water solubility, viscosity, and considerable resistance to enzymatic hydrolysis which resemble those of water-soluble dietary fiber. Since viscous-producing water-soluble dietary fiber reportedly slows absorption of dietary components such as glucose and cholesterol through hampering diffusion of the components in the intestine, ${ }^{14)}$ suppression of OVA penetration in the intestine by kefir might be mediated similarly by the viscous-producing property of kefiran. In fact, it has been reported that plasma

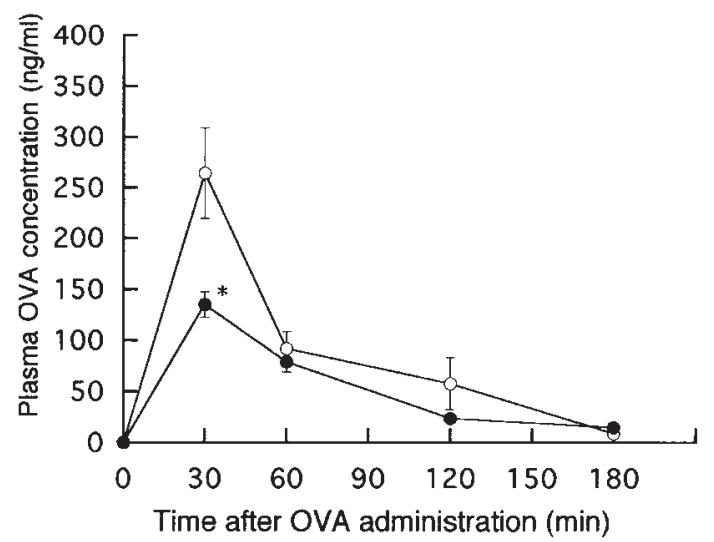

Fig. 1. Effect of Freeze-Dried Kefir on Intestinal Permeation of OVA in BALB/c Mice.

The time-course of changes in plasma OVA concentration after intragastric administration of OVA is shown. Open and closed circles represent OVA- (control) and OVA plus kefir-administered mice, respectively. Values are given as means \pm SEM of 6 mice per group. ${ }^{*}, p<0.05$ vs. control at each time point. concentrations of cholesterol and triglyceride were lowered by the consumption of kefiran in rats fed a cholesterol-enriched high fat diet, ${ }^{15)}$ and these results suggest that dietary kefiran suppresses lipid absorption in the small intestine. We are now investigating the effect of concomitant kefiran on OVA penetration in the intestinal loop of anesthetized mice. In addition, work is in progress to examine whether dietary kefir facilitates degradation of OVA, secretion of immunoglobulin A eliminating OVA, and/or the nonspecific mucosal barriers including mucus secretion in the intestine.

An obvious increase in anti-OVA IgG was observed 2 weeks after starting OVA administration in mice, and the levels were consistently lower in mice fed the kefir diet than those fed the control diet (Fig. 2A). Additionally, OVA-stimulated proliferations of splenic mononuclear cells ex vivo were significantly lower in mice fed

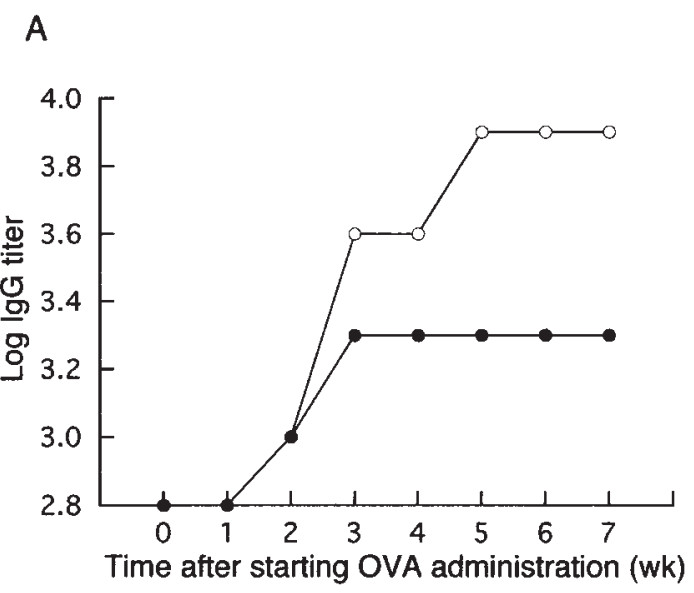

B

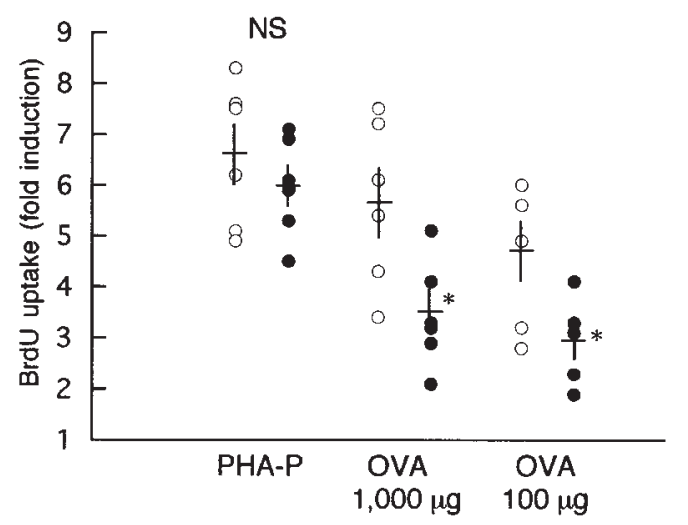

Fig. 2. Effect of Freeze-Dried Kefir on Oral Sensitization to OVA in $\mathrm{BALB} / \mathrm{c}$ Mice.

The time-course of changes in serum OVA-specific IgG titer (A) and proliferative response of splenic mononuclear cells ex vivo (B) are shown. Open and closed circles represent data on mice fed the control and kefir diets, respectively. In chart A, values for pooled samples of 6 mice per group are given. In chart B, results are shown as fold induction to values with no stimulant. Each value represents each mouse, and the horizontal and vertical bars show the means and SEM of 6 mice per group, respectively. ${ }^{*}, p<0.05$ vs. control. NS, not significant. 
the kefir diet than in those fed the control diet, while mitogen-stimulated proliferations were the same between the groups (Fig. 2B). These data suggest that oral sensitization to OVA was suppressed by dietary kefir in mice. Clearly, reduced penetration of antigen in the gastrointestinal tract can contribute to suppression of sensitization to the antigen. Therefore suppression of oral sensitization to OVA by dietary kefir would be at least partly mediated by the reduced intestinal penetration of the antigen observed in the present study. In addition, live active bacteria in kefir may participate in the suppression of oral sensitization. Because it has become accepted that commensal bacteria in the intestine are important in oral tolerance induction, ${ }^{16)}$ it is conceivable that lactic acid bacteria in kefir might be involved in the suppression of oral sensitization by inducing oral tolerance. In fact, the freeze-dried kefir used in the present study contained $1.4 \times 10^{7} \mathrm{cfu} / \mathrm{g}$ of lactic acid bacteria.

Taken together, the present findings suggest that freeze-dried kefir reduces intestinal permeation of food antigen, which contributes to suppression of oral sensitization. Therefore we propose that kefir is of use to prevent food allergy.

\section{Acknowledgments}

This work was supported financially by a Grantin-Aid for Scientific Research from the Ministry of Education, Culture, Sports, Science and Technology of Japan.

\section{References}

1) Saitoh, R., Yamaguchi, N., Sonoyama, K., and Kawabata, J., Effect of enzymatic modification of dietary wheat flour for reducing its allergenicity on oral sensitization to and intestinal absorption of ovalbumin. Biosci. Biotechnol. Biochem., 67, 2483-2485 (2003).

2) Tanabe, S., Tesaki, S., Watanabe, J., Fukushi, E., Sonoyama, K., and Kawabata, J., Isolation and structural elucidation of a peptide derived from edam cheese that inhibits b-lactoglobulin transport. J. Dairy Sci., 86, 464468 (2003).

3) Tesaki, S., Watanabe, J., Tanabe, S., Sonoyama, K., Fukushi, E., Kawabata, J., and Watanabe, M., An active compound against allergen absorption in hypoallergenic wheat flour produced by enzymatic modification. Biosci. Biotechnol. Biochem., 66, 1930-1935 (2002).

4) Watanabe, J., Fukumoto, K., Fukushi, E., Sonoyama, K., and Kawabata, J., Isolation of tryptophan as an inhibitor of ovalbumin permeation and analysis of its suppressive effect on oral sensitization. Biosci. Biotechnol. Biochem., 68, 59-65 (2004).

5) Aldinucci, C., Bellussi, L., Monciatti, G., Passali, G. C., Salerni, L., Passali, D., and Bocci, V., Effects of dietary yoghurt on immunological and clinical parameters of rhinopathic patients. Eur. J. Clin. Nutr., 56, 1155-1161 (2002).

6) Isolauri, E., Arvola, T., Sütas, Y., Moilanen, E., and Salminen, S., Probiotics in the management of atopic eczema. Clin. Exp. Allergy, 30, 1604-1610 (2000).

7) Kalliomäki, M., Salminen, S., Arvilommi, H., Kero, P., Koskinen, P., and Isolauri, E., Probiotics in primary prevention of atopic disease: a randomised placebocontrolled trial. Lancet, 357, 1076-1079 (2001).

8) Majamaa, H., and Isolauri, E., Probiotics: a novel approach in the management of food allergy. J. Allergy Clin. Immunol., 99, 179-185 (1997).

9) Rautava, S., Kalliomäki, M., and Isolauri, E., Probiotics during pregnancy and breast-feeding might confer immunomodulatory protection against atopic disease in the infant. J. Allergy Clin. Immunol., 109, 119-121 (2002).

10) Van de Water, J., Keen, C. L., and Gershwin, M. E., The influence of chronic yogurt consumption on immunity. J. Nutr., 129, 1492S-1495S (1999).

11) Reeves, P. G., Nielsen, F. H., and Fahey, G. C., Jr., AIN$93 \mathrm{G}$ purified diets for laboratory rodents: final report of the American Institute of Nutrition ad hoc writing committee on the reformulation of the AIN-76A rodent diet. J. Nutr., 123, 1939-1951 (1993).

12) Watanabe, J., Tanabe, S., Watanabe, M., Kasai, T., and Sonoyama, K., Consumption of hypoallergenic flour prevents gluten-induced airway inflammation in Brown Norway rats. Biosci. Biotechnol. Biochem., 65, 17291735 (2001).

13) La Riviere, J. W., and Kooiman, P., Kefiran, a novel polysaccharide produced in the kefir grain by Lactobacillus brevis. Arch. Mikrobiol., 59, 269-278 (1967).

14) Schneeman, B. O., and Tietyen, J., Dietary fiber. In "Modern Nutrition in Health and Disease" 8th ed., eds. Shills, M. E., Olson, J. A., and Shike, M., Lea and Febiger Philadelphia, Philadelphia, pp. 89-100 (1994).

15) Maeda, H., Zhu, X., Suzuki, S., Suzuki, K., and Kitamura, S., Structural characterization and biological activities of an exopolysaccharide kefiran produced by Lactobacillus kefiranofaciens WT-2B. J. Agric. Food Chem., 52, 5533-5538 (2004).

16) Tanaka, K., and Ishikawa, H., Role of intestinal bacterial flora in oral tolerance induction. Histol. Histopathol., 19, 907-914 (2004). 